\title{
Novo Modelo de Armadilha para Captura de Scolytinae e Platypodinae (Insecta, Coleoptera)
}

\author{
Acacio Geraldo de Carvalho ${ }^{1}$, Henrique Trevisan ${ }^{1}$
}

${ }^{1}$ Departamento de Produtos Floretais, Universidade Federal Rural do Rio de Janeiro - UFRRJ, Seropédica/RJ, Brasil

\section{RESUMO}

O monitoramento de insetos é atividade relevante no processo de avaliação da sanidade de povoamentos florestais, bem como em estudos ecológicos. Dessa forma, geralmente, os levantamentos são realizados utilizando-se armadilhas de captura. Em especial para os grupos Scolytinae e Platypodinae, são empregadas armadilhas de impacto, iscadas com álcool $96^{\circ} \mathrm{GL}$. Sendo assim, este trabalho tem o objetivo de descrever um novo modelo desse tipo de equipamento, denominado SEMIFUNIL. O projeto propõe o uso de materiais alternativos na fabricação da armadilha: garrafa PET de $2000 \mathrm{~mL}$, mangueira plástica, prato plástico e arame, buscando com isso redução de custos.

Palavras-chave: monitoramento de insetos, insetos xilófagos, entomologia florestal.

\section{A New Trap Model for Scolytinae and Platypodinae (Insecta, Coleoptera)}

\begin{abstract}
The insect monitoring is a relevant activity in the process of assessing forest stands health, as well as in ecological studies. Thus, in general, surveys are conducted using capture traps; for the Scolytinae and Platypodinae especially, groups impact traps are used, baited with alcohol $96^{\circ} \mathrm{GL}$. This paper aims to describe a new trap model, denominated SEMI-FUNNEL. The project suggests the use of alternative materials in the trap manufacturing: $2000 \mathrm{~mL}$ PET bottle, plastic hose, plastic plate and wire, thereby seeking the reduction of costs.
\end{abstract}

Keywords: insect monitoring, xylophagous insects, forest entomology. 
O estudo da ocorrência de insetos é prática importante no monitoramento da sanidade de povoamentos florestais, bem como em estudos ecológicos. Essa atividade, geralmente, é realizada utilizando-se armadilhas para captura de insetos (Carvalho, 1998).

Nesse contexto, as coleobrocas pertencentes às subfamílias Platypodinae e Scolytinae são consideradas, dentre os insetos monitorados em povoamentos florestais, os grupos mais relevantes. Embora no Brasil sejam pragas secundárias em reflorestamentos, proporcionam danos expressivos na madeira de toras recém-abatidas quando no campo expostas.

O aumento da população desse grupo de insetos em um povoamento florestal, que pode ser detectado pelo uso de armadilhas etanólicas, sugere que as árvores estão sob estresse, o que gera condições para a atração desses insetos. Nesse contexto, Penteado et al. (2011) relataram essa situação em reflorestamento de Azadirachta indica A. Juss (Meliaceae), tendo considerado Scolytinae como bioindicador do declínio das árvores.

Mediante ao exposto, entende-se que a armadilha etanólica, quando utilizada nesse contexto, é componente importante dessa atividade. Desse modo, implementar projetos desse tipo de equipamento, que proponham armadilhas baratas, eficientes e práticas, ou mesmo trabalhos que avaliem condições de uso desses equipamentos, são informações almejadas nas pesquisas que versam sobre o aprimoramento das etapas do monitoramento de insetos. Concernente a essas pesquisas, há trabalhos que propõem um modelo de armadilha que explora a atração primária de toras à Scolytinae (Flechtmann \& Gaspareto, 1997), armadilha etanólica (Berti-Filho \& Flechtmann, 1986), análises da influência da cor e da altura de instalação de armadilhas na captura de Scolytinae (Strom \& Goyer, 2001; Chen et al., 2010), projetos que propõem o uso de materiais reciclados na fabricação desses equipamentos (Moser \& Browne, 1978; Carvalho, 1998; Murari et al., 2012; Steininger et al., 2015) e, por fim, pesquisas que analisam a eficiência de captura de diferentes modelos de armadilha (Carrano-Moreira et al., 1994; Flechtmann et al., 2000).

No trabalho de Carvalho (1998), em que é descrito o projeto da armadilha Carvalho-47, fabricada, entre outros materiais, com uma garrafa PET, é apresentado o primeiro modelo brasileiro com esse tipo de material, cuja estrutura é toda feita com materiais reciclados. Iniciativa essa que viabilizou uma armadilha barata, prática e com eficiência comprovada em diversas pesquisas (Carvalho et al., 1996; Ferraz et al., 1999).

Diante dessa conjectura, este trabalho descreve o modelo de uma nova armadilha, denominada SEMIFUNIL. A construção desta armadilha é realizada utilizando-se materiais alternativos: garrafa PET de $2000 \mathrm{~mL}$, um pote com tampa plástica, prato plástico, mangueira de $5 \mathrm{~mm}$ de diâmetro por $31 \mathrm{~cm}$ de comprimento e arame, de acordo com as seguintes etapas:

1) Corte as porções correspondentes ao fundo e ao gargalo da garrafa, utilizando como guia ao corte a própria marcação presente na garrafa. O gargalo será transformado em funil coletor (Figura 1c). Descarte a porção do fundo e reserve o corpo da garrafa.

2) O corpo da garrafa, que nesta etapa apresenta-se como um segmento cilíndrico, deve ser cortado em duas partes simétricas. A marcação para realização do corte simétrico é obtida pelo achatamento do cilindro. Após o corte, posicione essas duas peças de costas uma para a outra,

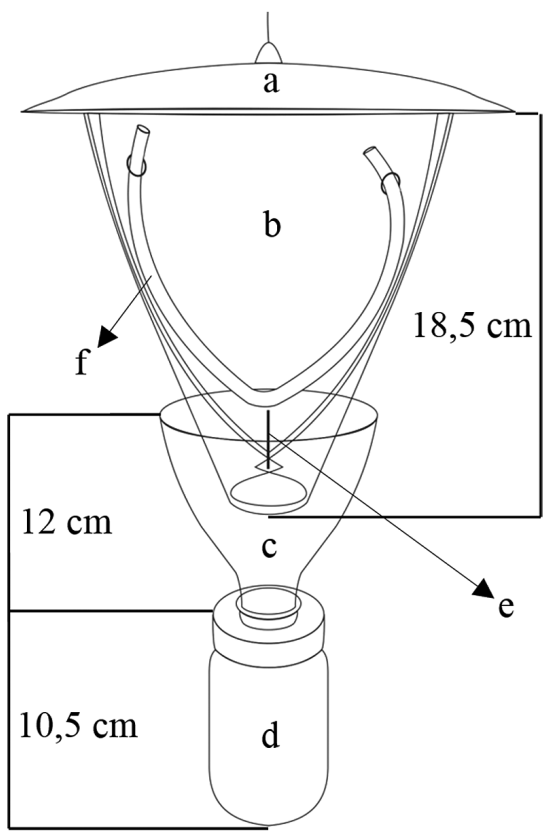

Figura 1. Vista frontal: (a) Prato protetor; (b) Painel interceptador "semifunil"; (c) Funil coletor; (d) Frasco armazenador; (f) Mangueira porta-isca; (e) Arame fixador.

Figure 1. Front view: (a) covering plate; (b) interceptor panel "Semi-funnel"; (c) collector funnel; (d) collecting bottle; (f) deposit of ethanol; (e) wire fastener. 
grampeando-as na extremidade superior. Após grampeamento, dobre as pontas da parte inferior de cada peça até que se cruzem. Utilizando um arame aquecido (Figura 1e), fure as pontas cruzadas, originando um "semifunil" na parte inferior de cada painel (Figura 1b).

3) Instale a mangueira plástica entre os dois painéis já fixados pelo grampeamento, de modo que uma ponta fique posicionada na parte frontal superior esquerda de um dos painéis, e a outra na parte frontal superior direita do outro painel. Ambas fixadas por orifício previamente feito em cada painel (Figuras 1f, 2f e 3).

4) O mesmo arame que origina os "semifunis" dos painéis serve para fixar ambos no funil coletor, funcionando como um eixo fixador (Figuras 1e, 2e e 3e). Esse eixo também é obtido pelo aquecimento do arame com posterior furação no funil coletor (Figura 3e).

5) Fure a tampa do frasco coletor utilizando um cano de metal aquecido, de dimensões suficientes

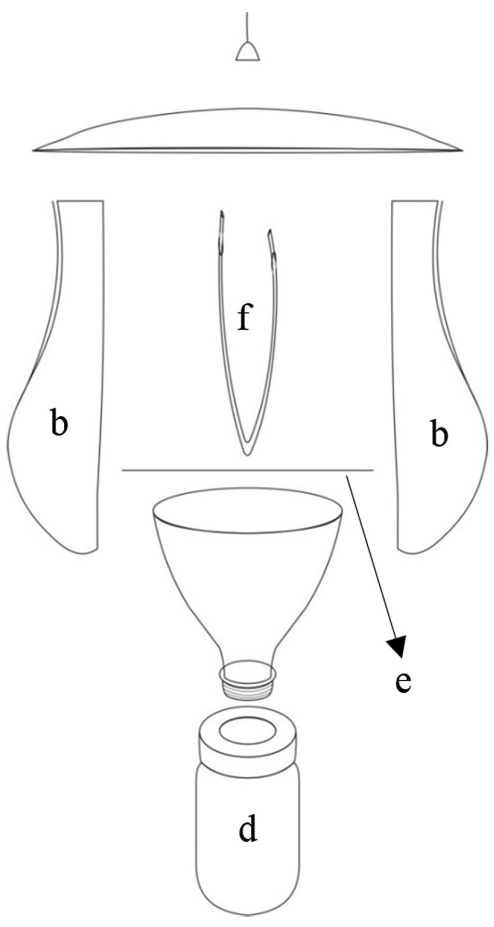

Figura 2. Vista lateral das peças: (b) Painel interceptador; (e) Arame fixador; (f) Mangueira porta-isca; (d) Frasco armazenador.

Figure 2. Side view of parts: (b) Interceptor panel; (e) wire fastener; (f) deposit of ethanol; (d) collecting bottle. para que o orifício produzido sirva de encaixe para a rosca do funil coletor (Figura 1d, 2d). Obs.: Deve-se usar a tampa da garrafa PET, tendo o fundo sido retirado previamente, como elemento de fixação da tampa do frasco coletor na rosca do funil coletor.

6) Fixe o prato plástico na parte superior da armadilha, utilizando-se de um arame flexível e de um orifício nele produzido (Figura 1a). $\mathrm{O}$ arame deve ser dobrado na parte superior do prato, garantindo assim sua amarração.

Para utilização da armadilha no campo, a mangueira deve ser abastecida com álcool 96 GL, adotando-se uma seringa para esse procedimento. No frasco armazenador, a mesma substância deve ser empregada como conservante para os insetos capturados. Recomendam-se amostragens semanais ou quinzenais para levantamentos de coleobrocas, renovando-se o álcool a cada coleta.

Avaliando a eficiência de captura de quatro armadilhas, Bossões (2011) registrou que os modelos SEMIFUNIL,

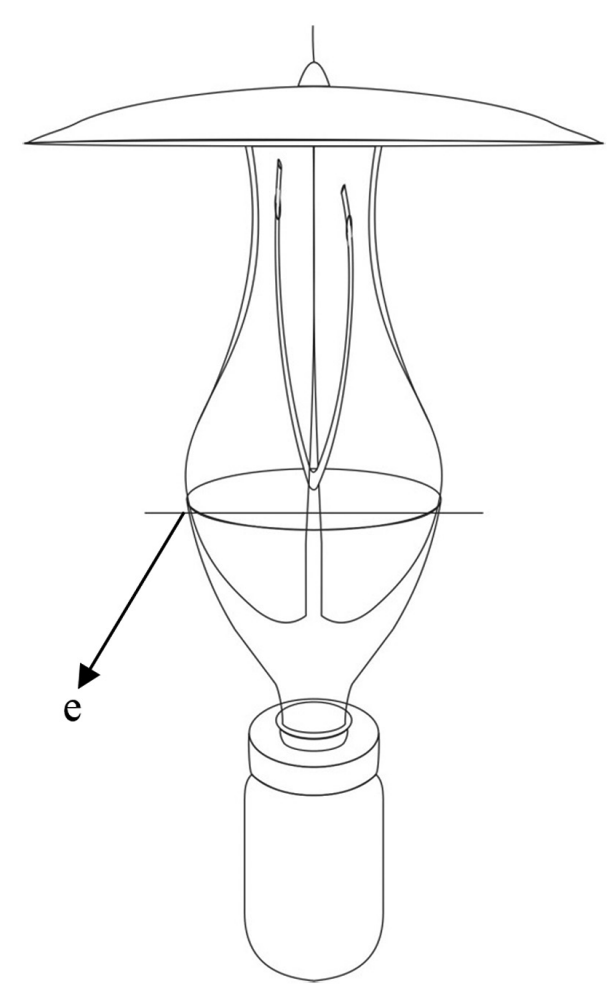

Figura 3. Vista lateral da armadilha: (e) Eixo fixador (arame rígido).

Figure 3. Side view the trap: (e) axis fastener (hard wire). 
Marques-Pedrosa, Carvalho 47 e Carvalho-47 adaptada, coletaram, em números percentuais: 39,$65 ; 24,91 ; 19,81$; e 15,6, respectivamente, do total de escolitíneos, em monitoramento realizado durante um ano. $\mathrm{O}$ autor relata ainda que o número médio de escolitíneos capturados pela armadilha SEMIFUNIL (47 \pm 53$)$, demonstrou-se igual estatisticamente quando comparado com os valores fornecidos pelas armadilhas Marques-Pedrosa $(30 \pm 25)$ e Carvalho-47 (24 \pm 24$)$, diferindo apenas

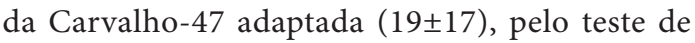
Kruskal-Wallis a 5\% de significância. Porém, o autor informa também que mesmo não possuindo a maior área interceptadora de insetos, $480 \mathrm{~cm}^{2}$, a armadilha SEMIFUNIL demonstrou-se com eficiência de captura análoga ao modelo Marques-Pedrosa, que possui maior área interceptadora, $1495 \mathrm{~cm}^{2}$, no entanto, não possui as vantagens de ser confeccionada com material reciclado e coletar menos resíduos. Em relação à comparação com o modelo Carvalho-47, Bossões (2011) ainda observou que embora a eficiência de captura também tenha sido similar estatisticamente ao equipamento descrito neste trabalho, a Carvalho-47 foi a única armadilha que forneceu valores médios estatisticamente iguais aos valores da armadilha Carvalho-47 adaptada, modelo este que se demonstrou estatisticamente menos eficiente na captura dos escolitíneos.

\section{STATUS DA SUBMISSÃO}

Recebido: 17 jul. 2014

Aceito: 24 jul. 2015

\section{AUTOR(ES) PARA CORRESPONDÊNCIA}

\section{Henrique Trevisan}

Departamento de Produtos Floretais, Universidade Federal Rural do Rio de Janeiro UFRRJ, BR 465, Km 07, Campus da UFRRJ, CEP 23890-000, Seropédica RJ, Brasil e-mail: hentrevisan@gmail.com

\section{REFERÊNCIAS}

Berti-Filho E, Flechtmann CAH. A model of ethanol trap to collect Scolytidae and Platypodidae (Insecta, Coleoptera). Ipef 1986; 34: 53-56.

Bossões RR. Avaliação e adaptação de armadilhas para captura de insetos em corredor agroflorestal [dissertação].
2011. Seropédica: Instituto de Biologia, Universidade Federal Rural do Rio de Janeiro.

Carrano-Moreira CF, Marques EM, Pedrosa-macedo JH. Eficiência de dois modelos de armadilhas de impacto e influência da altura na coleta de Scolytidae (Coleoptera). Revista Árvore 1994; 18(3): 256-264.

Carvalho AG, Rocha MP, Silva CAM, Lunz AM. Variação sazonal de Scolytidae (Coleoptera) numa comunidade de floresta natural de Seropédica, RJ. Floresta e Ambiente 1996; 3(2): 9-14.

Carvalho AG. Armadilha, modelo Carvalho-47. Floresta e Ambiente 1998; 5(1): 225-227.

Chen G, Zhang QE, Wang Y, Liu GN, Zhou X, Niu J et al. Catching Ips duplicatus (Sahlberg) (Coleoptera:Scolytidae) with pheromone-baited traps: optimal trap type, colour, height and distance to infestation. Pest Management Science 2010; 66(2): 213-219. PMid:19862793.

Ferraz FC, Carvalho AG, Coutinho CL, Souza NJ. Eficiência de armadilhas etanólicas para levantamento de coleópteros do reflorestamento de Eucalyptus citriodora em Pinheiral, RJ. Floresta e Ambiente 1999; 6(1): 159-162.

Flechtmann CAH, Gaspareto CL. A new trap for capturing Scolytidae (Coleoptera), based on primary attraction. Journal of Applied Entomology 1997; 121(1-5): 357-359. http://dx.doi.org/10.1111/j.1439-0418.1997.tb01419.x.

Flechtmann CAH, Ottati ALT, Berisford CW. Comparison of four trap types for ambrosia beetles (Coleoptera, Scolytidae) in Brazilian Eucalyptus stands. Journal of Economic Entomology 2000; 93(6): 1701-1707. http:// dx.doi.org/10.1603/0022-0493-93.6.1701. PMid:11142301.

Moser JC, Browne LE. A nondestructive trap for Dendroctonus frontalis Zimmerman (Coleoptera: Scolytidae). Journal of Chemical Ecology 1978; 4(1): 1-7. http://dx.doi.org/10.1007/ BF00988254.

Murari AB, Costa EC, Boscardin J, Garlet J. Modelo de armadilha etanólica de interceptação de voo para captura de escolitíneos (Curculionidae: Scolytinae). Pesquisa Florestal Brasileira 2012; 32(69): 115-117. http://dx.doi. org/10.4336/2012.pfb.32.69.115.

Penteado SRC, Carpanezzi AA, Neves MEJ, Santos ÁF, Flechtmann CAH. Escolitídeos como bioindicadores do "declínio do nim" no Brasil. Pesquisa Florestal Brasileira 2011; 31(65): 69-73. http://dx.doi.org/10.4336/2011. pfb.31.65.69.

Steininger MS, Hulcr J, Šigut M, Lucky A. Simple and efficient trap for bark and ambrosia beetles (Coleoptera: Curculionidae) to facilitate invasive species monitoring and citizen involvement. Journal of Economic Entomology 2015; 108(3): 1115-1123. http://dx.doi.org/10.1093/jee/ tov014. PMid:26470236.

Strom BL, Goyer RA. Effect of silhouette color on trap catches of Dendroctonus frontalis (Coleoptera: Scolytidae). Annals of the Entomological Society of America 2001; 94(6): 949-953. http://dx.doi.org/10.1603/0013-8746(2001)094[0948:EO SCOT]2.0.CO;2. 